\title{
Osteosarcoma of the Proximal Tibia: Limb-Sparing Resection and Reconstruction with a Modular Segmental Proximal Tibia Tumor Prosthesis
}

\author{
James C. Wittig, MD ${ }^{1}$, Camilo E. Villalobos, MD ${ }^{\mathbf{1}}$, Brett L. Hayden, MS ${ }^{\mathbf{1}}$, Ikjoon Choi, BS ${ }^{\mathbf{1}}$, \\ Andrew M. Silverman, $\mathbf{M S}^{1}$, and Martin Malawer, $\mathbf{M D}^{2}$ \\ ${ }^{1}$ Department of Orthopedic Surgery, The Mount Sinai Medical Center, New York, NY; ${ }^{2}$ Department of Orthopedic \\ Oncology, Washington Cancer Institute, Washington, DC
}

\begin{abstract}
A limb-sparing resection was performed for osteosarcoma of the proximal tibia in a young patient. A special modular segmental proximal tibial endoprosthesis that includes a hinged total knee component was used for reconstruction. A medial gastrocnemius rotational flap was utilized to reconstruct the extensor mechanism of the knee. The muscle flap aids in covering the prosthesis and helps to
\end{abstract}

protect against infection. Limb-sparing resection for tumors arising from the proximal tibia, and reconstruction with a modular segmental proximal tibia tumor prosthesis and gastrocnemius muscle flap, is a safe and reliable method for treating tumors involving this area.

Electronic supplementary material The online version of this article (doi:10.1245/s10434-010-1180-3) contains supplementary material, which is available to authorized users.

(C) Society of Surgical Oncology 2010

First Received: 27 July 2009;

Published Online: 7 July 2010

J. C. Wittig, MD

e-mail: Drjameswitting@aol.com 